\title{
Utilization of polymerase chain reaction on archival cytologic material: a comparison with fresh material with special emphasis on cerebrospinal fluids
}

\author{
Rubina Mattu르, Lynn Sorbara ${ }^{1}$, Armando C Filie ${ }^{1}$, Richard Little ${ }^{2}$, Wyndham Wilson ${ }^{3}$, \\ Mark Raffeld ${ }^{1}$ and Andrea Abati $^{1}$ \\ ${ }^{1}$ Laboratory of Pathology, Bethesda, MD, USA; ${ }^{2} H I V$ and AIDS Malignancy Branch, Bethesda, MD, USA and \\ ${ }^{3}$ Experimental Transplantation and Immunology Branch, National Cancer Institute/National Institutes of \\ Health, Bethesda, MD, USA
}

\begin{abstract}
Use of the polymerase chain reaction (PCR) for the detection of B- and T-cell clonality, Epstein-Barr virus (EBV) and Human Herpes Virus 8 (HHV 8) infection is gaining increasing importance as a diagnostic modality. These tests are usually performed on fresh specimens. There are instances when fresh material is not available and there is a clinical utility for the performance of PCR on archival material via slide scrape lysates (SSL). However, the suitability of archival material may be questioned. Records were searched for all archival cytology cases submitted for SSL molecular diagnostics tests since 1998. Results for each case were analyzed for PCR amplification status and individual test results. A randomly chosen control group of equivalent cytologic samples submitted fresh was evaluated for comparison of amplification status. In all, 241 PCR runs were performed on SSL of archival material from 112 cytologic samples (89 cerebrospinal fluids (CSFs), 13 fineneedle aspirates (FNAs), 10 effusions). Out of these samples, 95 (85\%) had amplifiable DNA, as assessed by a positive reaction for glyceraldehyde phosphate dehydrogenase (GAPDH). For the control group, 320 PCR runs were performed on 112 fresh cytologic samples (89 CSFs, 13 FNAs, 10 effusions). In total, 102 samples (91\%) had amplifiable DNA. There was no statistical difference in the amplification yield between the two groups $(P=0.2177)$. A morphologic review of 16 of the $17 \mathrm{SSL}$ archival cytologic cases that did not show amplification revealed 11/16 to be of sparse cellularity. Molecular diagnostic tests are performed routinely on fresh cytologic samples with excellent results. At times critical decisions on patient care may need to be made when fresh tissue is not available for molecular diagnostic tests. SSL of archival cytologic material can be used with excellent results for molecular diagnostic tests when fresh material is not available or when the cytologic diagnosis needs further clarification.

Modern Pathology (2004) 17, 1295-1301, advance online publication, 11 June 2004; doi:10.1038/modpathol.3800185
\end{abstract}

Keywords: cytology; slide scrape lysates; polymerase chain reaction

Molecular studies are now routinely performed in pathology laboratories for the diagnosis of various medical conditions such as cancer, infectious diseases and genetic disorders. ${ }^{1,2}$ One of the most commonly performed molecular tests is the polymerase chain reaction (PCR) which enables the amplification of specific sequences of nucleic acids from an extremely small amount of genetic starting material. ${ }^{3}$ In most laboratories, PCR is usually

Correspondence: Dr A Abati, MD, Cytopathology Section, Laboratory of Pathology, National Cancer Institute, Bldg. 10, Rm. 2A 19, 10 Center Drive, Bethesda, MD 20892, USA.

E-mail: abatia@mail.nih.gov

Received 23 February 2004; revised 23 April 2004; accepted 25 April 2004; published online 11 June 2004 performed on a variety of fresh specimens including blood, body fluids and tissues. Several small series have shown, however, that archival cytologic samples and formalin-fixed tissues may be suitable for evaluation by molecular tests. ${ }^{4-10}$ This principle may be extremely important when a diagnosis is unsuspected and when fresh tissue is no longer available, as well as for the testing of remotely obtained samples.

The utilization of molecular techniques on archival material via slide scrape lysates (SSL) for the diagnosis of lymphoma and leukemia is ideally suited for the characterization of cerebrospinal fluid (CSF). Although the positive identification of malignant hematopoietic cells within the CSF is of the utmost importance for diagnosis, staging and 
treatment, definitive diagnosis may be hindered based on overlapping cellular morphology. Viral infection or treatment effects may yield lymphoid cells that mimic a blast-like appearance. Conversely, genetically malignant cells may have unremarkable morphology. Several preliminary investigations of this problem have touted the use of molecular studies in tandem with conventional techniques for diagnostic enhancement in fresh and archival material. ${ }^{1,10-12}$

In contrast to fresh samples, archival material such as cytological specimens, paraffin-embedded, or frozen tissues, presents the opportunity for a careful morphologic review and interpretation prior to molecular analysis. This allows the trained cytologist to preselect cases and slide preparations for subsequent molecular analysis, resulting in optimal utilization and cost control in the molecular laboratory. As molecular techniques are improved and refined, diagnositic possibilities will become realities: recently, Hug et $a l^{13}$ demonstrated the use of single-cell PCR analysis of cells directly isolated from standard CSF cytospins for the diagnosis of B-cell malignancies.

Both clinicians and pathologists have operated under the assumption that fresh specimens have the best diagnostic yield for molecular studies. However, fresh material is not always available to perform molecular testing, and careful comparative studies are few. It is, therefore, important to assess whether archival tissues used as diagnostic samples will provide results comparable to that of fresh specimens.

Over the past 5 years, we have routinely performed molecular tests on archival cytologic material for diagnostic purposes. What initially began as an inquiry into a methods alternative, evolved into a vital diagnostic query performed on cases with ambiguous morphology. ${ }^{14}$ Our goal for this study was to determine if there were any differences in the degree of diagnostic utility for molecular studies (as measured by DNA amplification), between whole SSL of archival cytologic material as compared to freshly submitted cytology specimens, with particular emphasis on CSF.

\section{Materials and methods}

\section{Case Selection}

Results of 112 archival cytologic samples submitted for molecular diagnostic studies from July 1998 to July 2003, were generated via a computer search in the Molecular Diagnostics Unit of the National Cancer Institute, National Institutes of Health. These cases were obtained either through patient file reviews or from a previous study. ${ }^{14}$ The specimens (a mixture of Diff Quik and Papanicolaou-stained smears and cytospin preparations) included 89 cerebrospinal fluids, 13 fine-needle aspirations (FNAs) and 10 effusions. A majority of the samples were stained with Diff-Quik as this is the predominant stain used in our institution for CSFs and hematopoietic neoplasms.

Concurrently, for the control study specimens, case files from the same time period were reviewed for similar types of cytologic samples received fresh in the molecular diagnostics laboratory. Samples were selected consecutively until a preset number had been reached to reflect the exact numbers and proportions of the archival specimens: 89 CSFs, 13 FNAs and 10 effusions.

\section{DNA Isolation from Archival Cytospin and Smear Samples}

Coverslips were removed from the slides via immersion in xylene. A volume of $20 \mu \mathrm{l}$ of digestion buffer $(50 \mathrm{mM}$ Tris, $1 \mathrm{mM}$ EDTA, $0.5 \%$ Tween $\mathrm{pH}$ 8.0) was added to the slide and the surface was then scraped with a single edge razor blade (SSL). The cells/buffer mixture was transferred directly to a PCR tube. Volumes of 8-10 $\mu$ l of digestion buffer was again added to the slide and the procedure was repeated. Proteinase K ( $2 \mu \mathrm{l}$ of $10 \mathrm{mg} / \mathrm{ml})$ was added and the tubes were layered with mineral oil prior to incubation at $37^{\circ} \mathrm{C}$ for $60 \mathrm{~min}$ followed by $98^{\circ} \mathrm{C}$ for $10 \mathrm{~min}$. At the end of this incubation period, the samples were divided into PCR tubes containing $20 \mu \mathrm{l}$ of Gene Releaser resin (Bioventures, TN, USA). The tubes (with sample and resin) were preincubated in a thermocycler (Model 9700, Applied Biosystems, Foster City, CA, USA) according to the manufacturer's directions. At the end of the preincubation period, the appropriate master mixes were added to the tubes and 40 cycles of PCR were performed.

\section{DNA Isolation from Fresh Cerebral Spinal Fluid, FNA and Pleural Effusion Samples}

Fresh cerebral spinal fluid, FNA and pleural effusion samples were centrifuged at $1500 \mathrm{rpm}(450 \times g)$ for $10 \mathrm{~min}$. Supernatants were removed and the pellets were resuspended in digestion buffer (50 mM Tris, $1 \mathrm{mM}$ EDTA, 0.5\% Tween $\mathrm{pH}$ 8.0) with proteinase K. Samples were preincubated in a thermocycler (Model 9700, Applied Biosystems, Foster City, CA, USA) at $37^{\circ} \mathrm{C}$ for $60 \mathrm{~min}$ followed by $98^{\circ} \mathrm{C}$ for $10 \mathrm{~min}$. At the end of the preincubation period, the appropriate master mixes were added to the tubes and 40 cycles of PCR were performed.

\section{PCR Amplification and Detection of Products}

To test the amplification ability of each sample, PCR was performed with primers directed to the housekeeping gene, glyceraldehyde phosphate dehydrogenase (GAPDH) according previously described methods. ${ }^{15,16}$ Placental DNA was used as a positive control. 
For detection of Epstein-Barr Nuclear Antigen 2 (EBNA 2), latent membrane antigen-1 (LMP-1) and Human Herpes Virus-8 (HHV-8), PCR was performed using primers and methods described by Otsuki et al. ${ }^{17}$ Positive control DNA was isolated from the Raji and BC-1 cell lines, respectively.

For assays to detect viral components and GAPDH, amplification products were separated by $2 \%$ agarose gel electrophoresis and visualized with ethidium bromide staining. To increase sensitivity and selectivity of EBV viral genes, EBNA 2 detection, gel products were transferred to Nytran membranes and probed by Southern blot hybridization with a digoxigenin-labeled (DIG) internal probes. The posthybridization visualization was performed by chemiluminescence (Roche Diagnostics, Indianapolis, IN, USA).

To detect clonality of the immunoglobulin heavy chain gene $(I g H)$ and the T-cell receptor gamma gene, PCR was performed using primers and methods described by Segal et $a 1^{18}$ and McCarthy et $a{ }^{19}{ }^{19}$ respectively. DNA isolated from a blood sample of a patient with hairy cell leukemia was used as a positive (clonal) control for the $\mathrm{IgH}$ gene rearrangement studies. DNA isolated from the CEM and MOLT-4 cell lines were used as positive (clonal) controls for the T-cell receptor gene rearrangement studies. PCR products were separated by $16 \%$ polyacrylamide gel electrophoresis and visualized by ethidium bromide staining.

\section{Statistical Analysis}

Specimens were analyzed for DNA amplification status as assessed by a positive or negative reaction to GAPDH. The overall amplification yield for archival specimens was compared with that of fresh specimens to determine if there was any statistically significant difference between the two groups.

Specimens with no amplifiable DNA were not considered for further analysis. For those specimens which showed amplifiable DNA, results of specific molecular diagnostic tests were analyzed. These tests (Tables 1 and 2; Figures 1-3) included PCR for identification of viral infections (EBV and HHV-8) and PCR for clonality analysis of defined B- and T-cell populations. A total of 241 PCR runs (including GAPDH and 129 tests) were performed on microdissected archival specimens and 320 PCR runs (including GAPDH and 208 tests) on the fresh sample control group. Test results were evaluated for diagnostic yield. A comparison of the diagnostic yield for individual test results between the archival and fresh samples was analyzed.

The diagnostic samples were separately interpreted as either clonal or polyclonal (tests for $\mathrm{B}$ - and T-cell clonality) and as positive or negative (tests for analysis of viral DNA).

The statistical analysis was performed by using Fisher's exact test with Instat software (Graph pad).
Table 1 Molecular tests on archival cytologic samples

\begin{tabular}{lcccc}
\hline Test & $\begin{array}{c}\text { Number of } \\
\text { specimens }\end{array}$ & Polyclonal & Clonal & Nondiag \\
\hline IgH PCR & 24 & 11 & 4 & 9 \\
Framework III & 20 & 9 & 3 & 8 \\
Framework II & 4 & 2 & 1 & 1 \\
TCR gamma PCR & 12 & 7 & 5 & \\
Primer Jg 12 & 7 & 3 & 4 & \\
Primer Jp 12 & 5 & 4 & 1 & \\
Test & $\#$ & Positive & Negative & Nondiag \\
\hline EBV PCR & 86 & 16 & 70 & \\
EBNA-2 gene & 70 & 15 & 55 & \\
LMP-1 gene & 16 & 1 & 15 & \\
HHV-8 PCR & 7 & 1 & 6 & \\
ORF 26 gene & 7 & 1 & 6 & \\
\hline
\end{tabular}

Table 2 Molecular tests on fresh cytologic samples

\begin{tabular}{lrccc}
\hline Test & $\begin{array}{c}\text { Number of } \\
\text { specimens }\end{array}$ & Polyclonal & Clonal & Nondiag \\
\hline IgH PCR & 21 & 13 & 5 & 3 \\
Framework III & 21 & 13 & 5 & 3 \\
Framework II & 0 & 0 & 0 & \\
TCR gamma PCR & 16 & 4 & 10 & 2 \\
Primer Jg & 8 & 2 & 5 & 1 \\
Primer Jp p $_{12}$ & 8 & 2 & 5 & 1 \\
Test & $\#$ & Positive & Negative & Nondiag \\
\hline EBV PCR & 164 & 65 & 93 & 6 \\
EBNA-2 gene & 83 & 34 & 46 & 3 \\
LMP-1 gene & 81 & 31 & 47 & 3 \\
HHV 8 PCR & 7 & 3 & 4 & \\
ORF 26 gene & 7 & 3 & 4 & \\
\hline
\end{tabular}

\section{Results}

\section{Slide Scrap Lysates of Archival Cytologic Samples}

Overall, of the 112 SSL samples, 95 (85\%) showed amplifiable DNA as assessed by a positive reaction to GAPDH. Of the 17 specimens that did not show DNA amplification (including $16 \mathrm{CSF}$ and one FNA), 16 were available for study. A morphologic review of these cases revealed 11/16 to be of sparse cellularity, two were moderately cellular and two were highly cellular. With regard to diagnostic categories, four cases (25\%) were malignant, nine $(56 \%)$ showed atypical cells and three $(19 \%)$ were negative for malignant cells.

Amplifiable DNA was present in 73 of 89 CSF samples (82\%); 12 of 13 FNAs (92.3\%) and 10 of 10 effusions $(100 \%)$. Of those specimens with amplifiable DNA individual test results are summarized in Table 1. Overall, 120 of 129 tests analyzed (93\%) showed diagnostic results. Tests for B-cell clonality (IgH PCR) showed diagnostic results in 15 of 24 tests performed $(62.5 \%)$ and those for T-cell clonality 


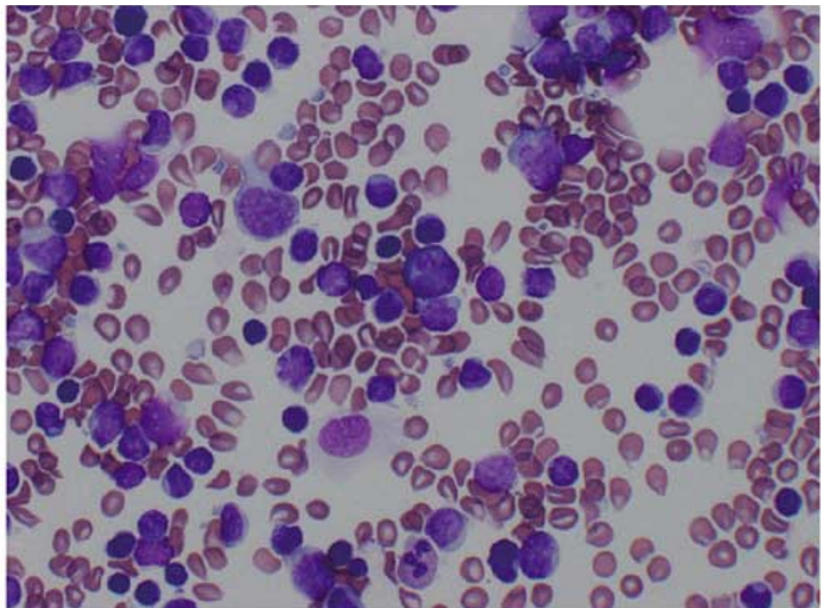

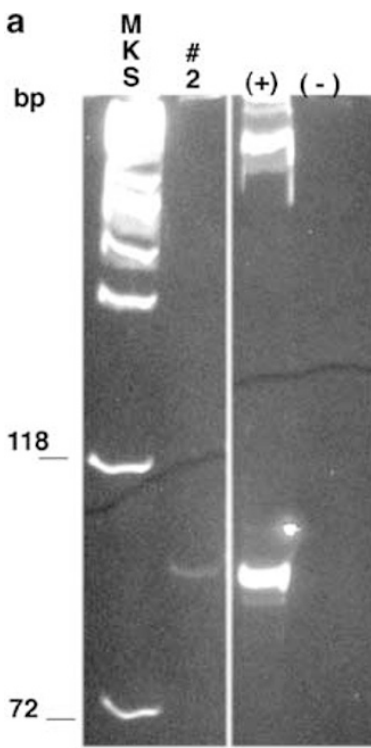

IGH FRIII

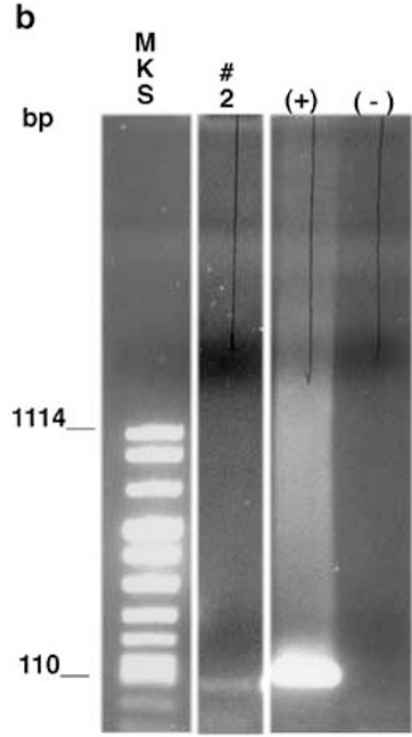

GAPDH

Figure 2 Immunoglobulin heavy chain gene rearrangement (IGH FRIII) and glyceraldehyde phosphate dehydrogenase (GAPDH) control gene amplification. (a) Cytospins of a CSF sample were used as template for JGH FRIII PCR using consensus primers directed to FRIII and the JH regions of the immunoglobulin heavy chain gene. Results show a faint but discrete band indicating a monoclonal process. (b) To ascertain amplification ability of the cytospin sample for case \#2, primers directed to the housekeeping gene, GAPDH were used for control PCR amplification. Results show a faint but discrete band indicating a positive reaction with a low number of cells. MKS $=$ DNA molecular weight marker; $(+)=$ positive control template for DNA amplification; $(-)=$ negative control (no template) for DNA amplification.

performed (100\%), as did those for LMP $1(16 / 16)$ and HHV 8 (7/7).

\section{Fresh Sample Submissions}

Overall, of the 112 samples, 102 (91\%) showed DNA amplification, as assessed by a positive reaction to GAPDH. Of 89 CSF samples, 81 (91\%); 12 of 13 FNA samples $(92.3 \%)$ and nine of 10 effusions (90\%) showed DNA amplification. The 10 specimens that did not show DNA amplification included eight CSFs, one FNA and one pleural fluid. A morphologic review of these cases revealed $5 / 10$ to be of sparse cellularity, two of moderate cellularity, two of high cellularity and one was unsatisfactory for evaluation. With regard to diagnostic categories, only one of the 10 cases $(10 \%)$ was malignant, while nine $(90 \%)$ were negative.

Of those specimens with amplifiable DNA, individual test results are summarized in Table 2 . Overall, 197 of 208 tests analyzed (94.7\%) showed diagnostic test results. Tests for B-cell clonality (IgH PCR) showed diagnostic results in 18 of 21 tests performed $(85.7 \%)$ and tests for T-cell clonality (TCR gamma PCR) showed diagnostic results in 14 of 16 tests $(87.5 \%)$. Viral DNA detection tests for EBNA 2 were diagnostic in 80 of 83 tests performed 


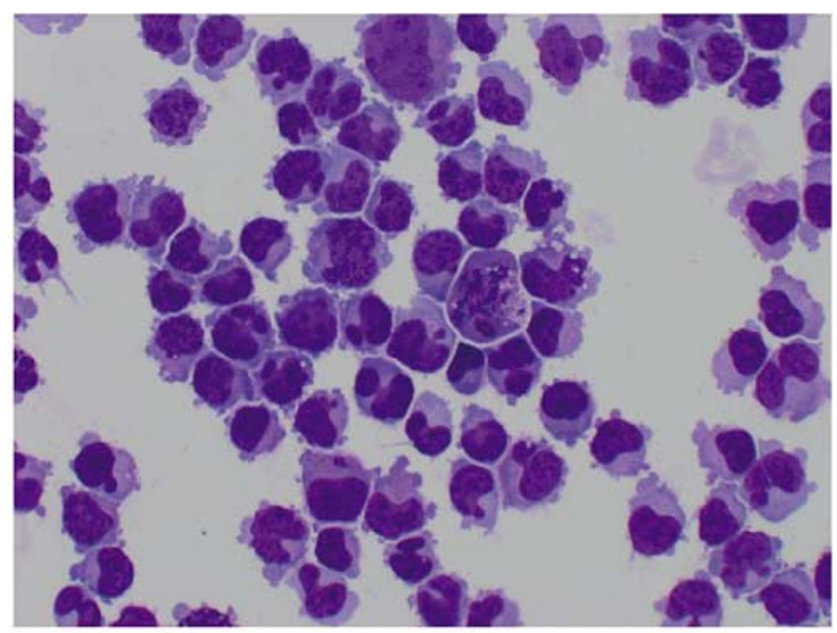

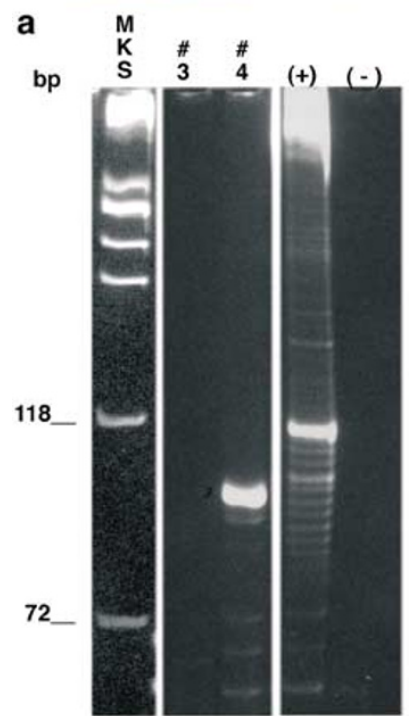

IGH FRIII b

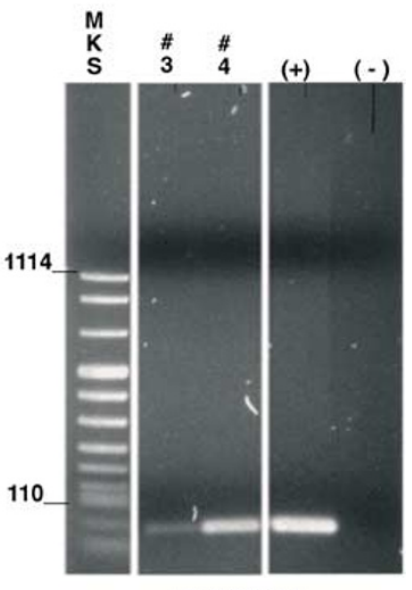

GAPDH
Figure 3 Immunoglobulin heavy chain gene rearrangement (IGH FRIII) and glyceraldehyde phosphate dehydrogenase (GAPDH) control gene amplification. (a) Cytospins of two separate CSF samples from the same patient (case \#3/\#4) were used as template as described in (2a). Amplification for the presence of a clone process was negative for case \#3 and strongly positive for case \#4. The cytospin for case \#4 reveals a monomorphous population of large, atypical lymphocytes. (Diff-Quik, $400 \times$ ). (b) Both case \#3 and \#4 show positive amplification with the housekeeping gene, $G A P D H$. MKS $=$ DNA molecular weight marker; $(+)=$ positive control template for DNA amplification; $(-)=$ negative control (no template) for DNA amplification.

(96.3\%); for LMP 1 in 78 of 81 (96.3\%) and HHV 8 in all seven tests.

\section{Statistical Analysis}

Statistical analysis showed no statistically significant difference in the yield of amplifiable DNA using archival and fresh cytologic specimens, overall $(P=0.2177)$. There was no statistically significant difference between the two groups irrespective of the specimen type evaluated; CSFs $(P=0.1232)$,
FNAs $(P=1.5200)$, and effusions $(P=1.0000)$. The diagnostic yield for individual test results between archival and fresh cytologic specimens showed no statistically significant difference for IgH PCR $(P=0.1011)$, TCR Gamma PCR $(P=0.4921)$, or EBNA PCR $(P=0.2505)$.

\section{Discussion}

Because the apparent utility of molecular testing has been established in certain diseases, the demand for this type of technology has increased proportionately. As results of molecular tests have become more refined, clinicians and pathologists alike have become dependent upon these tests, tolerating less and less of a possible diagnostic margin of error for their patients. This approach is particularly true in the diagnosis of a hematologic neoplasm involving the CSF where the addition of PCR-based studies has been shown to enhance diagnostic accuracy. ${ }^{1,10-12}$ This is evidenced through the results of the study by Rhodes et $a l,{ }^{12}$ which showed that $38 \%$ of cytologically negative CSFs were deemed positive by PCR studies. Conversely, $50 \%$ of the cytologically positive cases were confirmed by PCR with the other positive cases deemed 'equivalent' by PCR. These types of findings suggest a complementary approach to the evaluation of CSF for molecularly characterized malignancies such as lymphoma. The ability to visualize all of the material and then perform PCR greatly enhances the probability of reaching the correct diagnostic conclusion.

In the last few years, studies have appeared which have revealed the success of applying various molecular techniques to SSLs of cytologic samples. ${ }^{6,9,20-22}$ This has been achieved in small numbers with PCR tests for the diagnosis of lymphomas ${ }^{5,7,11,23}$ and also in a small number of CSF studies. ${ }^{10,14}$ In all references mentioned, the PCR tests performed on the cytologic material was found to provide important diagnostic information that was, in some instances, not possible by morphology alone. ${ }^{5,7,10-12}$ Archival specimens can, therefore, potentially be used in instances where no fresh tissue is easily available, when an unsuspected diagnosis is considered in the differential upon morphologic review and for retrospective testing of samples in patients who develop disease at a later date.

The use of PCR has on occasion been applied for definitive diagnosis of metastatic malignancies on FNA cytology specimens by comparing genetic abnormalities with the primary tumor. ${ }^{21,22}$ Very few studies, however, have actually compared the diagnostic yield of molecular tests performed on fresh and archival cytology specimens. One study compared recovery and amplification of mRNA for fresh and archival cervical smears. The archival samples included whole slide scrapings and microdissected epithelial cells. This study showed that RT-PCR was positive in all fresh and archival 
Table 3 Comparison of DNA amplification between archival and fresh cytologic samples

\begin{tabular}{lccc}
\hline Sample type & Number of samples & Amplification archival & Amplification fresh \\
\hline Overall & 112 & $95(85 \%)$ & $102(91 \%)$ \\
CSFs & 89 & $73(82 \%)$ & $81(91 \%)$ \\
FNAs & 13 & $12(92.3 \%)$ & $12(92.3 \%)$ \\
Effusions & 10 & $10(100 \%)$ & $9(90 \%)$ \\
\hline
\end{tabular}

samples and was not affected by type of fixative, processing method or microdissection. ${ }^{20}$

In another report, lymphoid tissue obtained by FNA was studied for detection of bcl2 oncogene. A total of 30 cases were examined. In 18 cases, DNA was obtained from either an archival Pap or a modified Giemsa-stained slide. In 12 cases, DNA was obtained using fresh specimens, either Diff Quikstained slide or needle rinse in RPMI. Both fresh and archival material provided sufficient DNA for PCR amplification and bcl2 detection. ${ }^{23}$ However, this study involved only a small number of cases for comparison of diagnostic yield between the two groups.

We conducted this study to compare the diagnostic yield of archival and fresh cytology specimens, particularly CSFs, using a much larger number of cases. A total of 112 cases of archival cytology specimens that included 89 CSFs, 13 FNAs and 10 effusions were analyzed for DNA amplification (Table 3). Similarly, we evaluated 112 matched random samples that were freshly submitted for molecular diagnostics including 89 CSFs, 13 FNAs and 10 effusions, as a control group. We found no statistically significant difference in DNA amplification between the two groups, in which $85 \%$ of archival SSL cases showed amplifiable DNA compared to $91 \%$ in the control group. In addition, no statistically significant difference was found in DNA amplification yield when CSFs, FNAs and effusion samples of the two groups were compared individually. Specimens with amplifiable DNA were also evaluated for diagnostic yield of different tests performed. These tests included IGH PCR, TCRG (gamma) PCR and EBNA-2 PCR. Once again, no statistically significant difference was seen when individual test results of fresh and archival material were compared.

In our study, we did not attempt to enrich the atypical cell populations by the application of microdissection techniques. Whether or not such a cell enrichment technology such as microdissection is necessary depends upon the sensitivity of the test, and the percentage of tumor cells in the population being studied. For example, as few as $30 \%$ of contaminating normal cells can mask LOH analyses. Conversely, the identification of clonal B- and T-cell populations can tolerate the presence of much higher percentages of contaminating (polyclonal) B- or $\mathrm{T}$ cells with sensitivities reaching $1-2 \%$ of tumor cells. The detection of viral sequences (such as EBNA2) or translocations routinely reach sensitivities of 1 in $10^{-4}-10^{-5}$ in polymorphous populations. Therefore, we believe that for the analyses described herein microdissection is not routinely necessary or needed.

In conclusion, our study establishes that there is no statistically significant difference in the diagnostic yield on a per sample basis between fresh and archival cytology specimens evaluated for molecular diagnostics. This study also emphasizes the potentially important role that PCR may play in archival cytologic specimens. This can be of particular importance when there is a limited amount of tissue for routine morphology and direct visualization, when the cytologic appearance is ambiguous or nondiagnostic, and for the study of remotely obtained samples. Our findings suggest the possibility of using a complementary approach to the evaluation of cytologic samples, particularly CSF, for molecularly characterized malignancies such as lymphoma. The ability to visualize all of the material and then perform PCR greatly enhances the probability of yielding the correct diagnosis.

\section{References}

1 Arribas JR, Clifford DB, Fichtenbaum CJ, et al. Detection of Epstein-Barr virus DNA in cerebrospinal fluid for diagnosis of AIDS-related central nervous system lymphoma. J Clin Microbiol 1995;33:1 580-1583.

2 Gessain A, Briere J, Angelin-Duclos C, et al. Human herpes virus 8 (Kaposi's sarcoma herpes virus) and malignant lymphoproliferations in France: a molecular study of 250 cases including two AIDS-associated body cavity based lymphomas. Leukemia 1997;11: 226-272.

3 Baumforth KR, Nelson PN, Digby JE, et al. Demystified ... the polymerase chain reaction. Mol Pathol 1999; 52:1-10.

4 Pan LX, Diss TC, Isaacson PG. The polymerase chain reaction in histopathology. Histopathology 1995;26: 201-217.

5 Alkan S, Lehman C, Sarago C, et al. Polymerase chain reaction detection of immunoglobulin gene rearrangement and bcl-2 translocation in archival glass slides of cytologic material. Diagn Mol Pathol 1995;4:25-31.

6 Apple SK, Hecht JR, Novak JM, et al. Polymerase chain reaction based K-ras mutation detection of pancreatic adenocarcinoma in routine cytology smears. Am J Clin Pathol 1996;105:321-326.

7 Grosso LE, Collins BT. DNA polymerase chain reaction using fine needle aspiration biopsy smears to evaluate non-Hodgkin's lymphoma. Acta Cytol 1999;43:837-841. 
8 Kikuchi M, Kitamura K, Nishio Y, et al. Diagnosis of B-cell lymphoma. Utility of the polymerase chain reaction for detecting clonality from archival cytologic smears. Acta Cytol 2002;46:349-356.

9 el Hamidi A, Kocjan G, Du MQ. Clonality analysis of archival cervical smears. Correlation of monoclonality with grade and clinical behavior of cervical intraepithelial neoplasia. Acta Cytol 2003;47:117-123.

10 Garicochea B, Cliquet MG, Melo N, et al. Leptomeningeal involvement in chronic lymphocytic leukemia identified by polymerase chain reaction in stored slides: a case report. Mod Pathol 1997;10:500-503.

11 Scrideli CA, Queiroz RP, Takayanagui OM, et al. Polymerase chain reaction on cerebrospinal fluid cells in suspected leptomeningeal involvement in childhood acute lymphoblastic leukemia: comparison to cytomorphological analysis. Diagn Mol Pathol 2003;12:124-127.

12 Rhodes CH, Glantz MJ, Glantz L, et al. A comparison of polymerase chain reaction examination of cerebrospinal fluid and conventional cytology in the diagnosis of lymphomatous meningitis. Cancer 1996;77:543-548.

13 Hug A, Storch-Hagenlocher B, Haas J, et al. Single-cell PCR analysis of the immunoglobulin heavy-chain CDR3 region for the diagnosis of leptomeningeal involvement of B-cell malignancies using standard cerebrospinal fluid cytospins. J Neurol Sci 2004;21:83-88.

14 Moses D, Sorbara L, Raffeld M, et al. Epstein-Barr virus in air-dried archival cerebrospinal fluid cytology: detection via conventional polymerase chain reaction. Mod Pathol 1999;12:49A.

15 Lin JC, Lin SC, De BK, et al. Precision of genotyping of Epstein-Barr virus by polymerase chain reaction using three gene loci (EBNA-2, EBNA-3C, and EBER): predominance of type A virus associated with Hodgkin's disease. Blood 1993;81:3372-3381 Erratum in: Blood 1993; 82:2268
16 Kingma DW, Weiss WB, Jaffe ES, et al. Epstein-Barr virus latent membrane protein-1 oncogene deletions: correlations with malignancy in Epstein-Barr virusassociated lymphoproliferative disorders and malignant lymphomas. Blood 1996;88:242-251.

17 Otsuki T, Kumar S, Ensoli B, et al. Detection of HHV-8/KSHV DNA sequences in AIDS-associated extranodal lymphoid malignancies. Leukemia 1996;10: 1358-1362.

18 Segal GH, Jorgensen T, Masih AS, et al. Optimal primer selection for clonality assessment by polymerase chain reaction analysis: I. Low grade B-cell lymphoproliferative disorders of nonfollicular center cell type. Hum Pathol 1994;25:1269-1275.

19 McCarthy KP, Sloane JP, Kabarowski JH, et al. A simplified method of detection of clonal rearrangements of the T-cell receptor-gamma chain gene. Diagn Mol Pathol 1992;1:173-179.

20 Chuaqui R, Cole K, Cuello M, et al. Analysis of mRNA quality in freshly prepared and archival Papanicolaou samples. Acta Cytol 1999;43:831-836.

21 Beaty MW, Zhuang Z, Park WS, et al. Fine-needle aspiration of metastatic clear cell carcinoma of the kidney: employment of microdissection and the polymerase chain reaction as a potential diagnostic tool. Cancer 1997;81:180-186.

22 Quezado MM, Abati AD, Albuquerque AV, et al. Morphologic diversity in malignant melanoma: the potential use of microdissection and the polymerase chain reaction for diagnosis. Mod Pathol 1998;11: 1010-1015.

23 Kube MJ, McDonald DA, Quin JW, et al. Use of archival and fresh cytologic material for the polymerase chain reaction. Detection of the bcl-2 oncogene in lymphoid tissue obtained by fine needle biopsy. Anal Quant Cytol Histol 1994;16:174-182. 